\title{
Mycobacterium tusciae sp. nov.
}

\author{
Enrico Tortoli, ${ }^{1}$ Reiner M. Kroppenstedt, ${ }^{2}$ Alessandro Bartoloni, ${ }^{3}$ \\ Giuseppe Caroli, ${ }^{4}$ Isabelle Jan, ${ }^{5}$ Jan Pawlowski ${ }^{6}$ and Stefan Emler ${ }^{5}$
}

Author for correspondence: Enrico Tortoli. Tel: +39055 4279199. Fax: +39 0554279292. e-mail: tortoli@dada.it

\author{
1 Laboratorio di \\ Microbiologia e Virologia, \\ Ospedale di Careggi, \\ 50139 Florence, Italy \\ 2 Deutsche Sammlung von \\ Mikroorganismen und \\ Zellkulturen $\mathrm{GmbH}, 38124$ \\ Braunschweig, Germany \\ 3 Cattedra di Malattie \\ Infettive, Università di \\ Firenze, 50121 Florence, \\ Italy \\ 4 Dipartimento di Salute \\ Pubblica e Biostatistica, \\ Università di Pisa, 56127 \\ Pisa, Italy \\ 5 Laboratoire Central de \\ Bactériologie, Hôpitaux \\ Universitaires de Genève, \\ 1211 Geneva, Switzerland \\ 6 Station de Zoologie, \\ Université de Genève, \\ 1224 Chene-Bougeries, \\ Switzerland
}

\begin{abstract}
A new, slow-growing, scotochromogenic mycobacterium was isolated from a lymph node of an immunocompromised child and subsequently from tap water and from a respiratory specimen of a patient with chronic fibrosis. Alcohol-acid-fastness, lipid patterns and the $G+C$ content clearly support the placement of this organism in the genus Mycobacterium. The isolates grew very slowly at temperatures ranging from 25 to $32{ }^{\circ} \mathrm{C}$ and showed activities of nitrate reductase, catalase, urease, arylsulfatase and Tween $\mathbf{8 0}$ hydrolysis. The organism was susceptible to all antimycobacterial drugs tested. The 16S rDNA sequence was unique and phylogenetic analysis placed the organism close to fast-growing species such as Mycobacterium farcinogenes, Mycobacterium komossense and Mycobacterium aichiense. These data support the conclusion that the isolates represent a new mycobacterial species, for which the name Mycobacterium tusciae sp. nov. is proposed. The type strain is strain Fl-25796'; a culture of this strain has been deposited in the DSMZ as strain DSM $44338^{\top}$.
\end{abstract}

Keywords: Mycobacterium tusciae sp. nov., taxonomy, identification, phylogeny

\section{INTRODUCTION}

Cervical lymphadenitis due to non-tuberculous (environmental) mycobacteria is encountered frequently in children. Mycobacterium scrofulaceum is classically considered to be the agent most frequently responsible for such disease, also known as scrofula, but in recent years an increasing prevalence of lymph-node infections due to Mycobacterium avium has been reported (Wolinsky, 1995).

Recently, several new scotochromogenic mycobacteria have been described as causes of paediatric cervical lymphadenitis (Haase et al., 1994; Springer et al., 1993; Tortoli et al., 1997). We feel that, in many pathologies attributed to $M$. scrofulaceum, this organism is likely to be only a scapegoat. The real causative agents are likely to be as yet undescribed mycobacteria, most of them characterized by common phenotypic features such as scotochromogenicity, grossly compatible with $M$. scrofulaceum.

The GenBank accession number for the $16 \mathrm{~S}$ rDNA sequence of strain $\mathrm{FI}$ $25796^{\top}$ is AF058299.
Attempts to identify unusual mycobacteria by conventional procedures may sometimes lead to misidentification, mainly because of the insufficient discriminatory power of current panels of biochemical and cultural features. The detection by HPLC of a new mycolic acid profile belonging to a mycobacterium isolated from a lymph node of a child was the first clue of a previously unreported species; this hypothesis was subsequently confirmed by more detailed investigations of its phenotypic and genotypic features. Results of taxonomic studies of this new organism, for which we propose the name Mycobacterium tusciae sp. nov., are reported here.

\section{METHODS}

Bacterial strains. In October 1995, a 7-year-old child who had been undergoing steroid therapy since the age of 3 , when a corticosteroid-susceptible nephrotic syndrome was diagnosed, was hospitalized because of a mild swelling on the left cheek. The lesion was aching and the overlying skin presented slightly reddish. An ultrasound scan revealed a round, regular-edged mass $(13 \times 11 \times 10 \mathrm{~mm})$ adhering to deep cutaneous tissues and characterized by inhomogeneous texture and oedema. After 3 weeks of treatment with 
amoxicillin/clavulanic acid, a mild reduction of the mass was noted and it was eventually removed by surgery 7 months later and so far no relapse has occurred. Strain FI$25796^{\mathrm{T}}$ was isolated from the excised lymph node, using Middlebrook 7H11 medium and conventional culture procedures (Nolte \& Metchock, 1995).

Two years later, during a local investigation for the presence of mycobacteria in drinking water, a further strain was isolated from tap water twice (FI-24797 and FI-27697) in a period covering several weeks. These isolations were made in a small town several dozens of kilometres away from the city where the child with lymphadenitis resides. Very recently, furthermore, a strain, probably lacking clinical significance, has been isolated in Switzerland from a respiratory specimen from a patient with chronic fibrosis.

Conventional identification methods. Colony morphology, pigment production in the dark and photoinduction and the ability to grow at temperatures ranging from 25 to $45^{\circ} \mathrm{C}$ were examined during a 6 week incubation on LowensteinJensen medium and Middlebrook 7H11 agar. Acid-alcoholfastness was determined by Ziehl-Neelsen and auramine $\mathrm{O}$ staining.

The following biochemical features (Kent \& Kubica, 1985) were investigated: niacin accumulation, nitrate reductase, arylsulfatase on days 3 and 14, semi-quantitative catalase, heat-stable catalase $\left(\mathrm{pH} 7,68^{\circ} \mathrm{C}\right)$, tellurite reductase, Tween 80 hydrolysis, $\beta$-glucosidase, acid phosphatase and urease. Inhibition tests included tolerance of thiophene-2carboxylic acid hydrazide, thiacetazone, $p$-nitrobenzoate, hydroxylamine, oleate, isoniazid and sodium chloride and growth on MacConkey agar without crystal violet.

Drug susceptibility. The macrodilution method in radiometric broth, recommended for $M$. avium (Siddiqi et al., 1993), was used in order to test the susceptibility of FI$25796^{\mathrm{T}}$ to ciprofloxacin, clarithromycin, ethambutol, rifabutin, rifampicin, sparfloxacin and streptomycin.

Lipid analyses. Mycolic acids of whole-organism methanolysates were investigated by TLC, as described previously (Minnikin et al., 1984), as well as by HPLC of bromophenacyl esters using a $\mathrm{C}_{18}$ Ultrasphere XL cartridge column (Beckman) on a System Gold instrument (Beckman) according to the standard procedure (Butler et al., 1992; Tortoli \& Bartoloni, 1996). Low and high molecular mass internal standards (Ribi; ImmunoChem) were added for identification of peaks.

Fatty-acid methyl esters, alcohols and mycolic-acid-cleavage products were obtained from $40 \mathrm{mg}$ wet biomass that was saponified, methylated and extracted as described previously (Miller, 1982). They were subsequently separated by GLC using a model 5898A gas chromatograph (Hewlett Packard). The Microbial IDENTIFICATION SySTEM software (Microbial ID) was used to identify the fatty acids.

Analysis of 16S rDNA. Extraction of DNA and amplification of the entire 16S rDNA gene were carried out as described previously (Kirschner et al., 1993). The 16S rDNA sequence was established by overlapping direct sequencing of both strands of the amplicons using fluorescence-labelled Dideoxy Dye terminators on an automated DNA sequencer (ABI 373A; Perkin-Elmer).

Determination of the DNA $\mathbf{G}+\mathbf{C}$ content. Nucleosides of isolated, hydrolysed and dephosphorylated DNA were separated by reverse-phase HPLC (Tamaoka \& Komagata, 1984) using a Nucleosil 100-5 $\mathrm{C}_{18}$ column. Nonmethylated lambda phage DNA with a G + C content of $49 \cdot 858 \mathrm{~mol} \%$ (Sanger et al., 1982) was used as a calibrator reference.

Phylogenetic analysis. The sequence of our strains was aligned with $16 \mathrm{~S}$ rDNA reference sequences from closely related mycobacterial species using the GENETIC DATA ENVIRONMENT software version 2.2 (Larsen et al., 1993). No gaps were removed, while probable sequencing errors within the reference sequence were omitted. A phylogenetic tree was constructed by using the neighbour-joining method (Saitou \& Nei, 1987). The method was applied to distances corrected for multiple hits and for unequal transition and transversion rates according to Kimura's two-parameter model (Kimura, 1980), omitting regions of uncertain alignment at both ends of the gene; tree positions were confirmed by parsimony analysis while bootstrapping was not applied due to the high similarity of mycobacterial $16 \mathrm{~S}$ rDNA.

\section{RESULTS}

\section{Morphological and cultural properties}

Cells of strains FI-25796 ${ }^{\mathrm{T}}$, FI-24797 and FI-27697 were acid-alcohol-fast, non-motile, non-spore-forming and rod-shaped. Growth was observed on Lowenstein-Jensen medium after 4 weeks incubation at temperatures ranging from 25 to $32{ }^{\circ} \mathrm{C}$; growth at $37^{\circ} \mathrm{C}$ was generally only observed on Middlebrook $7 \mathrm{H} 11$ agar and in radiometric Bactec 12B medium (Becton Dickinson), while inconsistent and scanty growth was observed on Lowenstein-Jensen medium at that temperature. Colonies were rough and scotochromogenic and characterized by a very elevated centre surrounded by a flat and uneven fringe. Growth was inhibited by the presence of $p$-nitrobenzoate, hydroxylamine, isoniazid, oleate or sodium chloride in culture media. No growth was obtained on MacConkey agar; however, the presence of thiophene2-carboxylic acid hydrazide and thiacetazone was tolerated.

\section{Biochemical properties}

The strains possessed nitrate reductase, heat-stable catalase, urease, acid phosphatase and arylsulfatase (positive at day 14 but not at day 3) and were able to hydrolyse Tween 80 . Accumulation of niacin and activities of $\beta$-galactosidase and $\beta$-glucosidase were not observed. Tellurite reductase was present in one of two environmental isolates.

\section{Antimicrobial susceptibility}

FI-25796 ${ }^{\mathrm{T}}$ was fully susceptible in vitro to all antimicrobials tested, while the inhibitory efficacy of ethambutol was limited. Minimal inhibitory concentrations were (in $\mu \mathrm{g} \mathrm{ml} \mathrm{m}^{-1}$ ): ciprofloxacin $\leqslant 1$, clarithromycin $\leqslant 0.5$, ethambutol 8 , rifabutin $\leqslant 0.5$, rifampicin $\leqslant 0.5$, sparfloxacin $\leqslant 0.5$ and streptomycin $\leqslant 2$. 
Table 1. Comparison of the mycolic acid patterns of strain $\mathrm{Fl}-25796^{\top}$ and other slow-growing mycobacteria

\begin{tabular}{|c|c|c|c|c|c|}
\hline Taxon & $\alpha$-Mycolates & $\alpha^{\prime}$-Mycolates & Methoxymycolates & Ketomycolates & Wax esters \\
\hline FI-25796 & + & - & - & + & + \\
\hline M. bohemicum & + & - & - & + & + \\
\hline M. conspicuum & + & - & - & + & + \\
\hline M. cookii & + & + & - & - & + \\
\hline M. gordonae & + & - & + & + & - \\
\hline$M$. interjectum & + & - & - & + & + \\
\hline$M$. lentiflavum & + & + & - & + & - \\
\hline M. scrofulaceum & + & - & - & + & + \\
\hline M. szulgai & + & - & + & + & - \\
\hline M. xenopi & + & - & - & + & + \\
\hline
\end{tabular}

Table 2. Fatty acid content of strain FI-25796'

\begin{tabular}{|lc|}
\hline Fatty acid & Content (\%) \\
\hline $12: 0$ & $0 \cdot 22$ \\
$14: 0$ & $5 \cdot 23$ \\
$15: 0$ & $0 \cdot 31$ \\
$16: 1$ cis 7 & $1 \cdot 05$ \\
$16: 1$ cis 8 & $1 \cdot 14$ \\
$16: 1$ cis 9 & $6 \cdot 72$ \\
$16: 0$ & $29 \cdot 86$ \\
$16: 0$ 8-methyl & $0 \cdot 19$ \\
$18: 0$ alcohol & $11 \cdot 33$ \\
$17: 0$ & $0 \cdot 35$ \\
$18: 1$ cis & $0 \cdot 50$ \\
$18: 1$ cis 9 & $21 \cdot 39$ \\
$18: 1$ cis 11 & $0 \cdot 53$ \\
$18: 0$ & $1 \cdot 83$ \\
$18: 0$ 10-methyl & $10 \cdot 49$ \\
$20: 0$ alcohol & $8 \cdot 28$ \\
$20: 0$ & $0 \cdot 30$ \\
\hline
\end{tabular}

\section{Lipid analysis}

TLC analysis detected wax esters and $\alpha$ - and ketomycolates in FI-25796 ${ }^{\mathrm{T}}$, a pattern shared by a number of scotochromogenic, slow-growing mycobacteria, e.g. Mycobacterium bohemicum, Mycobacterium conspicuum, Mycobacterium hiberniae, Mycobacterium interjectum, M. scrofulaceum and Mycobacterium xenopi (Table 1).

Analysis of fatty acids revealed a pattern typical of mycobacteria (Table 2), composed of unbranched, saturated and unsaturated fatty acids plus tuberculostearic acid (10-methyl $\left.\mathrm{C}_{18 ; 0}\right)$. Eicosanol, which is a cleavage product of the wax ester, was also found. The fatty acid profile of FI-25796 ${ }^{\mathrm{T}}$ was indistinguishable from those of Mycobacterium aurum and $M$. scrofulaceum.

From HPLC analysis, a mycolic acid pattern characterized by only one early cluster of peaks (Fig. 1) was found for the three isolates, which did not show

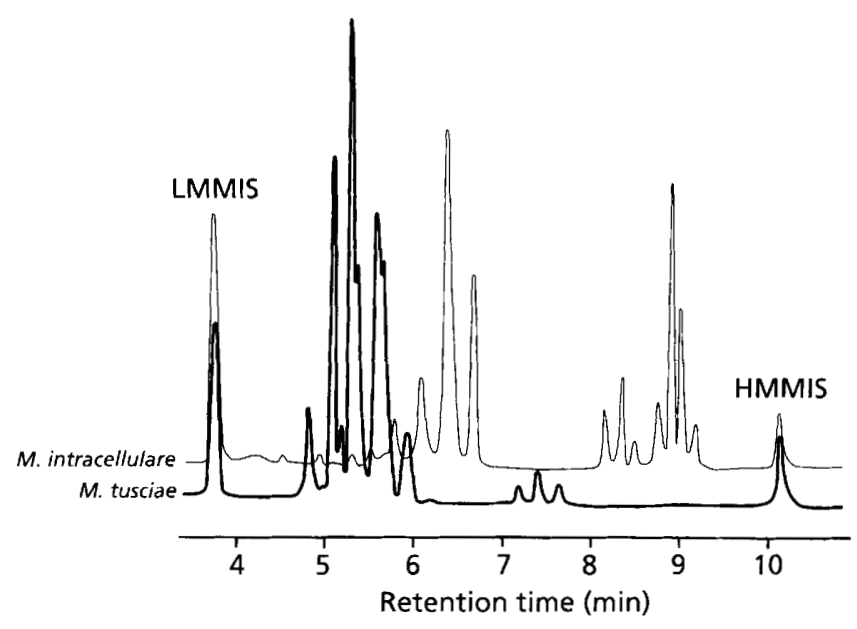

Fig. 1. Mycolic acid pattern of $M$. tusciae strain $\mathrm{FI}-25796^{\mathrm{T}}$ obtained by HPLC analysis compared with that of Mycobacterium intracellulare. LMMIS, Low-molecular-mass internal standard; HMMIS, high-molecular-mass internal standard.

significant similarity to any other previously reported mycobacterial mycolic acid profile.

\section{G + C content}

The $\mathrm{G}+\mathrm{C}$ content of DNA of FI-25796 ${ }^{\mathrm{T}}$ was 66.4 $\mathrm{mol} \%$.

\section{5 rDNA sequence analysis}

DNA sequences of 1445 bp from PCR-amplified $16 \mathrm{~S}$ rDNA of strains FI-25796 ${ }^{\mathrm{T}}$, FI-24797 and FI-27697 yielded a unique, as yet undescribed sequence for a mycobacterial species. In contrast to the growth behaviour in culture, the sequence pattern was characteristic of a fast-growing mycobacterium, showing the deletion of a stretch of 12 nucleotides between positions 413 and 425 within helix 18 (alignment with Mycobacterium tuberculosis reference sequence MTU16SRN from GenBank). 


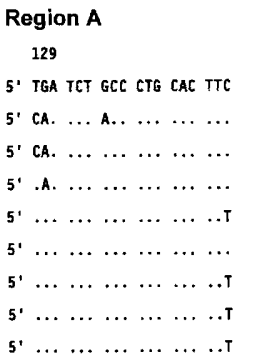

177 CGG ATA GG-ACCA CGG GAT GCA TGT CT- TGT GGT GGA $\ldots \ldots \ldots \ldots$. . . AA ..C ... ... ... .C. ... . .

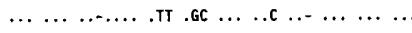
$\ldots \ldots \ldots \ldots, \pi, G \in \ldots \ldots$ ......... ... .C. ... ..G TG- ... А.... ..... т.-... т.. AC. т.. C.G T. ........ .. A ... tTCC.TA tT. . .TC ... ..G . . T G.. A.G ... .. . .. CACC.TG .T. .TC ... ..G . CT G.. A.G .. ... ... тАсCT.. T.. .C. ... ..G T. . .. . . .. M. tuberculosis (MTU16SRN) M. avium (MAV16SRN) M. scrofulaceum (MSC16SRN) M. simiae (MSI16SRN) M. farcinogenes (MFAR16S) M. komossense (MKOM16S) M. flavescens (MFL16SRN) M. smegmatis (MSM16SRN) Strain Fl-25796

\section{Region B}

533

5. CCT CTT TCA CCA TCG ACG AAG G-- IC CGG GTT CTC TCG GAT TGA CGG TAG GTG GAG AAG AAG CAC CGG $M$. tuberculosis (MTU16SRN)

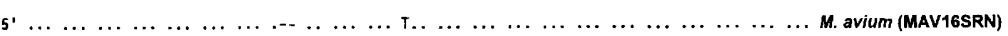

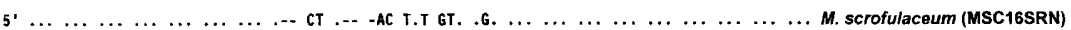

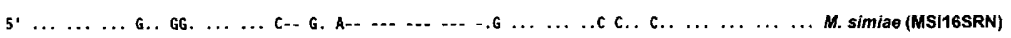

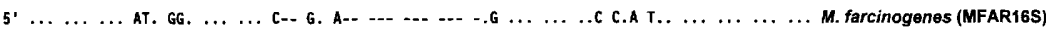

5 ' ........ GT. GG. ... ... C.- G. A-. ................ C.A T.. . . . . . . M. komossense (MKOM16S)

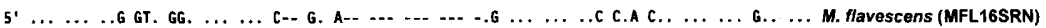

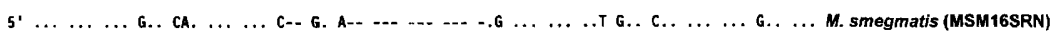

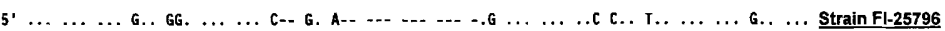

Fig. 2. Selected mycobacterial sequences of hypervariable regions $A$ and $B$ within the 165 rDNA. Positions, as derived from the $E$. coli sequence (GenBank reference sequences), are indicated. Only nucleotides that differ from $M$. tuberculosis are shown; dashes indicate deletions.

\section{Phylogenetic analysis}

Within the first variable region (A) of the $16 \mathrm{~S}$ rDNA, the signature sequence of the novel species was different in several positions from all published mycobacterial sequences; the second variable region (B), within helix 18 , resembled fast-growing species as well as the slow-growing species of the group of $\mathrm{Myco-}$ bacterium simiae (Fig. 2). Phylogenetic analysis grouped the novel species close to Mycobacterium aichiense, Mycobacterium komossense and Mycobacterium farcinogenes and to a new non-pathogenic mycobacterial species, strain PAH135 (Wang et al., 1995). A phylogenetic tree has been established (Fig. 3) showing the position of the new species with regard to fast-growing mycobacteria and representative slowgrowing mycobacteria. The tree was rooted using Nocardia asteroides as an outgroup. The position of the new species has been confirmed by parsimony analysis, while bootstrap values have been omitted due to the high degree of similarity of $16 \mathrm{~S} \mathrm{rDNA}$ sequences in mycobacteria.

\section{Differentiation of the novel species from other related mycobacteria}

Strains FI-25796 ${ }^{\text {T }}$, FI-24797 and FI-27697 are slowgrowing, scotochromogenic mycobacteria that can be distinguished from other related mycobacteria as follows: from $M$. xenopi by nitrate reduction, Tween hydrolysis, urease and inability to grow at $45^{\circ} \mathrm{C}$; from $M y c o b a c t e r i u m$ gordonae by nitrate reduction, semiquantitative catalase and urease; from $M$. scrofulaceum by nitrate reduction, semi-quantitative catalase, Tween 80 hydrolysis and urease; from Mycobacterium szulgai by semi-quantitative catalase, urease and $\beta$ glucosidase; from Mycobacterium lentiflavum by nitrate reduction and Tween 80 hydrolysis; from $M$. interjectum by nitrate reduction; from Mycobacterium flavescens by semi-quantitative catalase and urease;

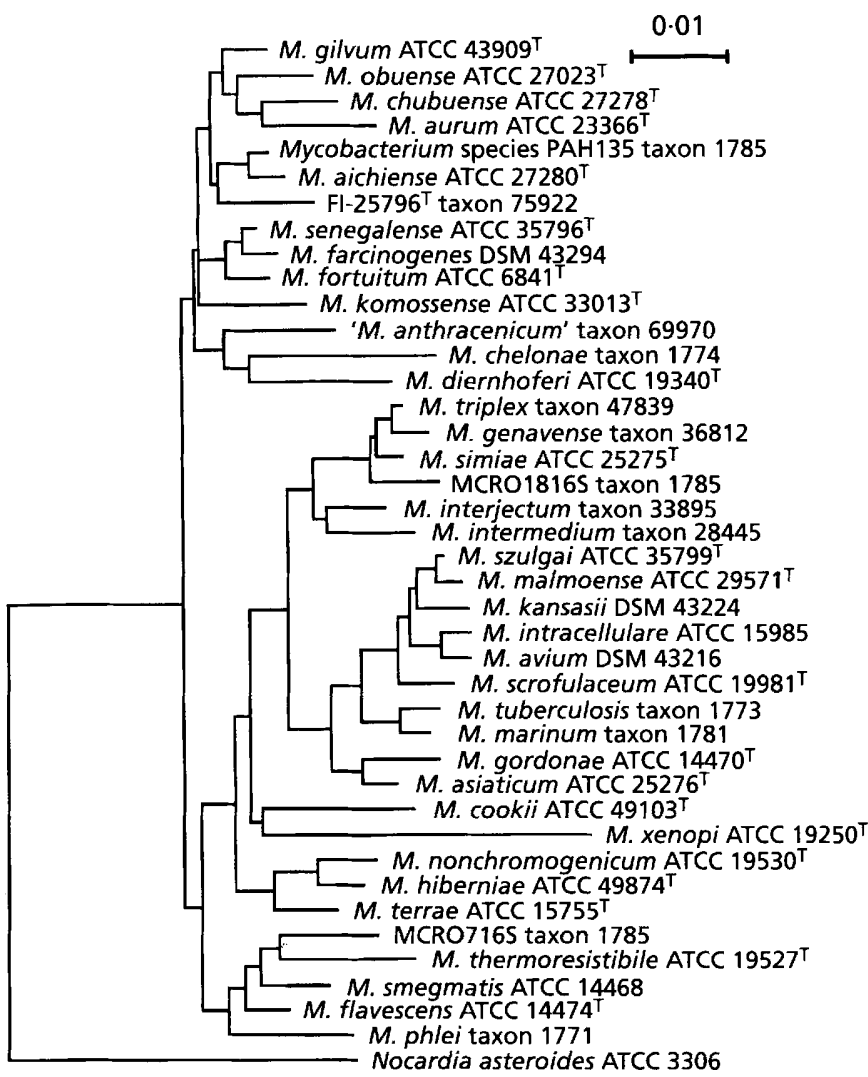

Fig. 3. Phylogenetic tree constructed using the neighbourjoining method and rooted with Nocardia asteroides as an outgroup.

and from $M$. bohemicum by nitrate reduction and Tween 80 hydrolysis (Table 3). Furthermore, the HPLC profile differs macroscopically from that of each of the aforementioned species. The $16 \mathrm{~S}$ rDNA sequence yields a characteristic pattern that differenti- 
Table 3. Selected distinguishing characteristics of strain Fl-25796 ${ }^{\top}$

Characters that distinguish strain FI-25796 ${ }^{\mathrm{T}}$ from other scotochromogenic, slow-growing mycobacterial species are shown. Date for other taxa were taken from Reischl et al. (1998), Springer et al. (1993, 1996) and Wayne \& Kubica (1986). Reactions are scored as: - negative; + , positive; or $\mathrm{v}$, variable.

\begin{tabular}{|c|c|c|c|c|c|c|c|c|c|}
\hline Characteristic & FI-25796 ${ }^{\mathrm{T}}$ & M. gordonae & M. xenopi & M. scrofulaceum & M. szulgai & M. lentiflavum & M. flavescens & M. interjectum & M. bohemicum \\
\hline Nitrate reduction & + & - & - & - & + & - & + & - & - \\
\hline Catalase over $45 \mathrm{~mm}$ & - & + & - & + & + & v & + & $\mathrm{v}$ & - \\
\hline Tween 80 hydrolysis & + & + & - & - & $\mathrm{v}$ & - & + & v & - \\
\hline Tellurite reduction & $\mathrm{v}$ & - & $\mathrm{v}$ & $\mathrm{v}$ & $\mathrm{v}$ & $\mathrm{v}$ & v & + & - \\
\hline Arylsulfatase $(3 \mathrm{~d})$ & - & - & $\mathrm{v}$ & - & - & - & + & - & - \\
\hline Urease & + & - & - & + & + & $\mathrm{v}$ & - & $\mathrm{v}$ & + \\
\hline$\beta$-Glucosidase & - & - & - & - & + & - & - & - & - \\
\hline $\mathrm{NaCl}$ tolerance & - & - & - & - & - & - & + & - & - \\
\hline Growth at $25^{\circ} \mathrm{C}$ & + & + & - & + & + & + & + & + & $\mathrm{v}$ \\
\hline Growth at $45^{\circ} \mathrm{C}$ & - & - & + & - & - & - & - & - & - \\
\hline
\end{tabular}

ates strains FI-25796 ${ }^{\mathrm{T}}$, FI-24797 and FI-27697 from any other previously described species.

\section{DISCUSSION}

Non-tuberculous mycobacteria are a major cause of cervical lymphadenitis in pre-school children. The presence of such organisms in soil, dust, food and tame animals, coupled with the propensity of children to put potentially contaminated objects in their mouths, suggest that oral mucosae, and particularly gingivae during tooth eruption, may be common portal of entry. The detection in tap water of a mycobacterium identical to the one isolated from a cervical lymph node may well support such a hypothesis.

The isolation from a sterile site is usually considered to be a sufficient criterion for the clinical relevance of a non-tuberculous mycobacterium (Wallace et al., 1990). $M$. tusciae, isolated from a child's lymph node, therefore seems to be potentially pathogenic, at least for immunocompromised individuals like the one described here, who had undergone steroid treatment.

The present organism appears to be differentiable from other slow-growing, scotochromogenic mycobacteria by conventional tests (Table 3), without significant variations among the strains isolated so far. As for the contribution of lipid analysis to the recognition of $M$. tusciae, while HPLC yielded a unique fatty acid profile (Fig. 1), the mycolic acid pattern that was obtained by TLC (Table 1) was shared by various scotochromogenic species and the fatty acid composition (Table 2) was indistinguishable from those of $M$. aurum and M. scrofulaceum.

Sequence analysis of the $16 \mathrm{~S}$ rDNA gene plays an important role in the description of new mycobacterial species. At the phylogenetic level, our isolate is characterized by a position intermediate between slowand fast-growing mycobacteria, as inferred by the presence of a short helix 18 within the hypervariable region $\mathrm{B}$ of the $16 \mathrm{~S}$ rDNA gene (Stahl \& Urbance, 1990). Other slow-growing mycobacteria ( $M$. simiae, Mycobacterium genavense, Mycobacterium inter- medium, $M$. interjectum) are characterized by a similar molecular marker but, in contrast to FI-25796 ${ }^{\mathrm{T}}$, FI24797 and FI-27697, they all share an identical sequence within hypervariable region $B$. Thus, the novel species represents a new entity of a slow-growing mycobacterium with phylogenetic characteristics of fast-growing species.

While phylogenetically closely related, fast-growing species such as $M$. flavescens and Mycobacterium senegalense are only known to cause infections in animals (Hamid et al., 1991; Mohan, 1985), other species, such as $M$. aichiense, $M$. komossense and a new polycyclic-aromatic-hydrocarbon-degrading species, PAH135 (Wang et al., 1995; Grosser et al., 1991), are not known to be pathogenic for humans. Its possible pathogenicity therefore emphasizes the need for specific recognition of $M$. tusciae.

\section{Description of Mycobacterium tusciae sp. nov.}

Mycobacterium tusciae (tu'sci.ae. L. gen. n. tusciae referring to the Italian region of Tuscany, where all the organisms were isolated).

Cells are alcohol-acid-fast and rod-shaped. At temperatures between 25 and $32^{\circ} \mathrm{C}$, scotochromogenic colonies grow in about 4 weeks on Lowenstein-Jensen medium. Growth at $37^{\circ} \mathrm{C}$ is inconsistent and requires a longer incubation, while no growth occurs at $42^{\circ} \mathrm{C}$. Colonies are rough and strongly yellow-pigmented. Their early microscopic morphology on Middlebrook $7 \mathrm{H} 11$ agar is characterized by a very elevated centre surrounded by an uneven flat fringe. Positive for nitrate reductase, heat-stable catalase, Tween 80 hydrolysis after $10 \mathrm{~d}$ incubation, arylsulfatase after 14 but not after $3 \mathrm{~d}$ incubation and urease, but negative for niacin and $\beta$-glucosidase. $M$. tusciae produces less than $45 \mathrm{~mm}$ of foam in a semi-quantitative catalase test. Growth is inhibited on MacConkey agar and by addition to the culture medium of sodium chloride, $p$ nitrobenzoate, hydroxylamine, isoniazid or oleate, but not by thiophene-2-carboxylic acid or thiacetazone. The strain is susceptible to ciprofloxacin, clarithromycin, rifabutin, rifampicin, sparfloxacin and 
streptomycin. Lipid composition is characterized by the presence of $\alpha$ - and ketomycolates and wax esters. The HPLC profile of mycolic acid bromophenacyl esters is unique. The fatty acid pattern is composed of hexadecanoic acid $(30 \%)$, hexadecenoic acids $(9 \%)$, octadecanoic acid $(2 \%)$, octadecenoic acids $(22 \%)$ and tuberculostearic acid $(10 \%)$. Two alcohols, $\mathrm{C}_{18: 0}$ $(11 \%)$ and $\mathrm{C}_{20: 0}(8 \%)$, are also found. The $\mathrm{G}+\mathrm{C}$ content of the DNA is $66.4 \mathrm{~mol} \%$. The $16 \mathrm{~S}$ rDNA sequence demonstrates that strains FI-25796 ${ }^{\mathrm{T}}$, FI24797 and FI-27697 belong to a novel species phylogenetically related to $M$. aichiense and $M$. farcinogenes, with characteristics of a fast-growing mycobacterium; the signature sequence within region $\mathrm{A}$ is characteristic of M. tusciae, however. Strain FI-25796 ${ }^{\mathrm{T}}$ is the type strain of Mycobacterium tusciae and has been deposited in the DSMZ as strain DSM $44338^{\mathrm{T}}$.

\section{ACKNOWLEDGEMENTS}

We thank W. R. Butler (CDC, Atlanta, USA) for valuable advice and L. Galassi (Istituto di Igiene, Università di Firenze, Firenze, Italy) for providing information concerning environmental isolates. The work of S. E. was supported by a grant from the Fondation Lancardis du Centre Valaisan de Pneumologie.

\section{REFERENCES}

Butler, W. R., Thibert, L. \& Kilburn, J. O. (1992). Identification of Mycobacterium avium complex strains and some similar species by high-performance liquid chromatography. J Clin Microbiol 30, 2698-2704.

Grosser, R. J., Warshawsky, D. \& Vestal, J. R. (1991). Indigenous and enhanced mineralization of pyrene, benzo[a]pyrene, and carbazole in soils. Appl Environ Microbiol 57, 3462-3469.

Haase, G., Skopnik, H., Bätge, S. \& Böttger, E. C. (1994). Cervical lymphadenitis caused by Mycobacterium celatum [letter]. Lancet 344, 1020-1021.

Hamid, M. E., Mohamed, G. E., Abu-Samra, M. T., el-Sanousi, S. M. \& Barri, M. E. (1991). Bovine farcy: a clinico-pathological study of the disease and its aetiological agent. J Comp Pathol 105, 287-301.

Kent, P. T. \& Kubica, G. P. (1985). Public Health Mycobacteriology. A Guide for the Level III Laboratory. Atlanta: US Department of Health and Human Services.

Kimura, M. (1980). A simple method for estimating evolutionary rates of base substitutions through comparative studies of nucleotide sequences. $J$ Mol Evol 16, 111-120.

Kirschner, P., Springer, B., Vogel, U., Meier, A., Wrede, A., Kiekenbeck, M., Bange, F. C. \& Böttger, E. C. (1993). Genotypic identification of mycobacteria by nucleic acid sequence determination: report of a 2-year experience in a clinical laboratory. J Clin Microbiol 31, 2882-2889.

Larsen, N., Olsen, G. J., Maidak, B. L., McCaughey, M. J., Overbeek, R., Macke, T. J., Marsh, T. L. \& Woese, C. R. (1993). The ribosomal database project. Nucleic Acids Res 21, 3021-3023.

Miller, L. T. (1982). Single derivatization method for routine analysis of bacterial whole-cell fatty acid methyl esters, including hydroxy acids. $J$ Clin Microbiol 16, 584-586.
Minnikin, D. E., Minnikin, S. M., Parlett, J. H., Goodfellow, M. \& Magnusson, M. (1984). Mycolic acid patterns of some species of Mycobacterium. Arch Microbiol 139, 225-231.

Mohan, K. (1985). Mycobacterium senegalense from bovines in Eastern Nigeria. J Appl Bacteriol 59, 277-281.

Nolte, F. S. \& Metchock, B. (1995). Mycobacterium. In Manual of Clinical Microbiology, pp. 400-437. Edited by P. R. Murray, E. J. Baron, M. A. Pfaller, F. C. Tenover \& R. H. Yolken. Washington, DC: American Society for Microbiology.

Reischl, U., Emler, S., Horak, Z., Kaustova, J., Kroppenstedt, R. M., Lehn, N. \& Naumann, L. (1998). Mycobacterium bohemicum sp. nov., a new slow-growing scotochromogenic mycobacterium. Int J Syst Bacteriol 48, 1349-1355.

Saitou, N. \& Nei, M. (1987). The neighbor-joining method: a new method for reconstructing phylogenetic trees. Mol Biol Evol 4, 406-425.

Sanger, F., Coulson, A. R., Hong, G. F., Hill, D. F. \& Petersen, G. B. (1982). Nucleotide sequence of bacteriophage lambda DNA. $J$ Mol Biol 162, 729-773.

Siddiqi, S. H., Heifets, L. B., Cynamon, M. H., Hooper, N. M., Laszlo, A., Libonati, J. P., Lindholm-Levy, P. J. \& Pearson, N. (1993). Rapid broth macrodilution method for determination of MICs for Mycobacterium avium isolates. J Clin Microbiol 31, 2332-2338.

Springer, B., Kirschner, P., Rost-Meyer, G., Schröder, K. H., Kroppenstedt, R. M. \& Böttger, E. C. (1993). Mycobacterium interjectum, a new species isolated from a patient with chronic lymphadenitis. J Clin Microbiol 31, 3083-3089.

Springer, B., Wu, W. K., Bodmer, T. \& 10 other authors (1996). Isolation and characterization of a unique group of slowly growing mycobacteria: description of Mycobacterium lentiflavum sp. nov. J Clin Microbiol 34, 1100-1107.

Stahl, D. A. \& Urbance, J. W. (1990). The division between fastand slow-growing species corresponds to natural relationships among the mycobacteria. J Bacteriol 172, 116-124.

Tamaoka, J. \& Komagata, K. (1984). Determination of DNA base composition by reverse-phase high-performance liquid chromatography. FEMS Microbiol Lett 25, 125-128.

Tortoli, E. \& Bartoloni, A. (1996). High-performance liquid chromatography and identification of mycobacteria. Rev Med Microbiol 7, 207-219.

Tortoli, E., Kirschner, P., Springer, B. \& 7 other authors (1997). Cervical lymphadenitis due to an unusual mycobacterium. Eur $J$ Clin Microbiol Infect Dis 16, 308-311.

Wallace, R. J., Jr, O'Brien, R., Glassroth, J., Raleigh, J. \& Dutt, A. (1990). Diagnosis and treatment of disease caused by nontuberculous mycobacteria. Statement of the American Thoracic Society, prepared by an ad hoc committee of the Scientific Assembly of Microbiology, Tuberculosis, and Pulmonary Infection. Am Rev Respir Dis 142, 940-953.

Wang, R. F., Cao, W. W. \& Cerniglia, C. E. (1995). Phylogenetic analysis of polycyclic aromatic hydrocarbon degrading mycobacteria by 16 S rRNA sequencing. FEMS Microbiol Lett 130 , $75-80$.

Wayne, L. G. \& Kubica, G. P. (1986). The Mycobacteria. In Bergey's Manual of Systematic Bacteriology, vol. 2, pp. 1435 1457. Edited by P. H. A. Sneath, N. S. Mair, M. E. Sharpe \& J. G. Holt. Baltimore: Williams \& Wilkins.

Wolinsky, E. (1995). Mycobacterial lymphadenitis in children: a prospective study of 105 nontuberculous cases with long-term follow-up. Clin Infect Dis 20, 954-963. 